\title{
SOFT-FEEDBACK OMP FOR THE RECOVERY OF DISCRETE-VALUED SPARSE SIGNALS
}

\author{
Susanne Sparrer, Robert F.H. Fischer \\ Institute of Communications Engineering, Ulm University, 89081 Ulm, Germany \\ Email: susanne.sparrer@uni-ulm.de,robert.fischer@uni-ulm.de
}

\begin{abstract}
In Compressed Sensing, a real-valued sparse vector has to be reconstructed from an underdetermined system of linear equations. However, in many applications of digital communications the elements of the unknown sparse vector are drawn from a finite set. The standard reconstruction algorithms of Compressed Sensing do not take this knowledge into account, hence, enhanced algorithms are required to achieve optimum performance. In this paper, we propose a new approach for the reconstruction of discrete-valued sparse signals. On the one hand, the algorithm is tailored to the discrete nature of the signal. On the other hand, reliability information is utilized within the successive reconstruction procedure. Via numerical simulations it is shown that the proposed variant of the Orthogonal Matching Pursuit clearly outperforms the wellknown standard versions.
\end{abstract}

\section{INTRODUCTION}

In a number of communication scenarios the noisy receive vector $\boldsymbol{y}$ at one time instance is given by ${ }^{1}$

$$
\boldsymbol{y}=\boldsymbol{A} \boldsymbol{x}+\boldsymbol{n},
$$

where $\boldsymbol{x}$ is the transmit vector of dimension $L$ with symbols either zero or drawn from the set $\mathcal{C} \stackrel{\text { def }}{=}\{ \pm 1\}, A \in \mathbb{R}^{K \times L}$, $K \ll L$, is the measurement matrix which, in a communication scenario can be identified with the channel, and $\boldsymbol{n}$ is the i.i.d. zero-mean Gaussian noise vector with variance $\sigma_{\mathrm{N}}^{2}$ per component. This setting corresponds to an underdetermined observation of $\boldsymbol{x}$ through $\boldsymbol{y}$. However, the transmit vector is assumed to be sparse, i.e., only $s \ll K$ of the $L$ elements are \pm 1 (active), the remaining $L-s$ are zero. We always assume the sparsity $s$ to be fixed and known.

At the receiver, to recover the vector $\boldsymbol{x}$ given $\boldsymbol{y}$, the following optimization problem has to be solved $\left(\mathcal{C}_{0} \stackrel{\text { def }}{=} \mathcal{C} \cup\{0\}\right)$

$$
\hat{\boldsymbol{x}}=\underset{\tilde{\boldsymbol{x}} \in \mathcal{C}_{0}^{L}}{\operatorname{argmin}}\|\boldsymbol{A} \tilde{\boldsymbol{x}}-\boldsymbol{y}\|_{2}^{2} \quad \text { with } \quad\|\tilde{\boldsymbol{x}}\|_{0}=s .
$$

This work was supported by Deutsche Forschungsgemeinschaft (DFG) under grant FI 982/8-1.

${ }^{1}$ Notation: $\|\cdot\|_{p}$ denotes the $\ell_{p}$ norm. $a_{k}$ is the $k^{\text {th }}$ column vector of the matrix $\boldsymbol{A}$, and $a_{l m}$ is the element in the $l^{\text {th }}$ column and $m^{\text {th }}$ row of $\boldsymbol{A}$. $\boldsymbol{A}_{\mathcal{S}}$ is the matrix composed of the columns of $\boldsymbol{A}$, whose indices are in the set $\mathcal{S}$, and $\boldsymbol{x}_{\mathcal{S}}$ is the vector with the elements of $\boldsymbol{x}$, whose indices are in the set $\mathcal{S} . \overline{\mathcal{S}}$ is the complement of the set $\mathcal{S}$ w.r.t. $\{1, \ldots, L\} . \boldsymbol{A}^{\top}$ and $\boldsymbol{A}^{+}$denote the transpose and the Moore-Penrose (left) pseudoinverse of $\boldsymbol{A}$, respectively. $\mathcal{Q}_{\mathcal{C}}(\cdot)$ : element-wise quantization to a given alphabet $\mathcal{C} \cdot \operatorname{Pr}\{\cdot\}$ : probability; $\mathrm{E}\{\cdot\}$ : expectation.
Thus, a discrete-valued sparse vector has to be estimated from an underdetermined set of linear equations.

Such a discrete compressed sensing problem is known in many fields of digital communications, e.g., in sensor networks, where $L$ sensors with low activity transmit data independently and a fusion center with $K$ antennas has to reconstruct the signals transmitted by the sensors [1]. Other examples are peak-to-average power reduction in orthogonal frequency-division multiplexing [2], the detection of pulsewidth-modulated signals in radar applications [3], and source coding [4].

Due to sparsity and the discrete nature of $\boldsymbol{x}$, problem (2) is nonconvex, even if relaxed to $\ell_{1}$-minimization. In the literature, different solutions based on compressed sensing (CS) [5] are known. First, there are several extensions to the simplex algorithm like the Branch-and-Bound and the CuttingPlane algorithm [6] for solving the $\ell_{1}$-minimization problem. Both algorithms require the simplex algorithm to run multiple times and have a prohibitively high computational complexity which restricts their use to very small dimensionalities.

Second, the problem can be solved by cascading a standard CS algorithm (ignoring the discrete nature of the symbols) with a lattice decoder [7], e.g., the sphere decoder (SD) [8]. While the CS algorithm, e.g., OMP [9] or CoSaMP [10], is used only for the detection of the support set, the SD reconstructs the discrete values at the support positions and can even detect support positions that have been erroneously chosen by the CS algorithm. Although the computational complexity of this approach is much smaller than the one of the previously mentioned algorithms, the average computational complexity of the SD grows with increasing noise level.

In this paper, an enhanced reconstruction algorithm for discrete sparse signals based on the OMP is proposed. On the one hand, the discrete nature of the symbols is explicitly taken into account and, on the other hand, reliability information is utilized within the successive reconstruction procedure. In each iteration of the OMP, a soft estimate of $\boldsymbol{x}$ is calculated based on a suitable error model. Although the results are shown for OMP, the principle idea can be generalized for related greedy algorithms such as CoSaMP and DThresh [11]. Compressed Sensing utilizing prior knowledge on the active symbols is rarely available in the literature, a Bayesian approach has been presented in [12]. However, in this paper, we take on a communications perspective and hence apply tools and measures from digital communications to successfully solve the Compressed Sensing recovery task. 
The paper is organized as follows. In Sec. 2, after a short introduction into the state-of-the-art OMP, we derive an OMP variant with soft feedback. The adjustment of the required parameters is discussed in Sec. 3. A comparison of the performance of the proposed algorithm to the standard OMP via numerical simulations is given in Sec. 4, followed by brief conclusions in Sec. 5.

\section{FINITE-ALPHABET OMP}

In this section, after a short introduction into the general principle of the OMP algorithm, the new algorithm is derived.

\subsection{Straightforward Versions of OMP}

First, we discuss the conventional OMP and its straightforward application to discrete-valued signals. The pseudocode representation is given in Alg. 1 (Variant A, i.e., only the lines tagged by an A are active).

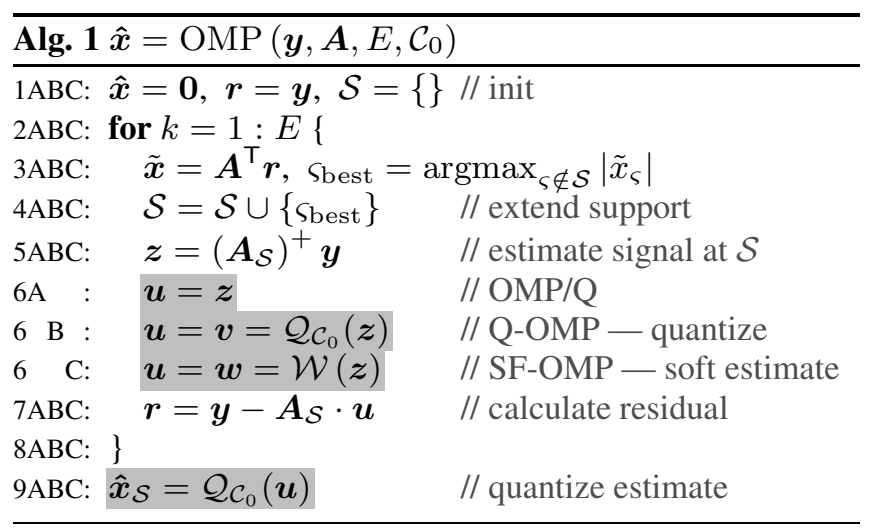

In each iteration, a new element is added to the estimated support set $\mathcal{S}$ in a greedy fashion. Then, the signal at the support positions, $\hat{\boldsymbol{x}}_{\mathcal{S}}$, is estimated via the Moore-Penrose pseudoinverse. Based thereon, the residual is updated. The OMP stops after $E \geq s$ iterations [7,13]. Since the elements of $\boldsymbol{x}$ are known to be drawn from the finite set $\mathcal{C}_{0}$, the elements of the estimated vector $\hat{\boldsymbol{x}}$ can simply be quantized to $\mathcal{C}_{0}$ subsequent to the decoding procedure. ${ }^{2}$ We denote this decoder, consisting of OMP with successive quantization, by OMP/Q.

In OMP/Q, the knowledge about the discrete nature of the signal is only used after the decoding, while the actual signal estimation procedure is still based on real numbers as in conventional OMP. An alternative strategy is to embed the quantization inside the iterative algorithm; we denote this strategy as Q-OMP [14]. Hence, the signal is immediately quantized after the estimation step (Line 6 B, Alg. 1). The calculation of the residual is carried out with respect to the quantized signal estimate. The pseudocode representation is also given in Alg. 1, Variant B.

In the language of digital communications, hard decisions are taken within this decoder, hence hard feedback is employed. As long as the decisions are correct, an improved

\footnotetext{
${ }^{2}$ If the sparsity is known at the decoder, which is assumed throughout the paper, the quantizer may include this knowledge.
}

performance can be expected. However, in case of decision errors, erroneous symbols are fed back leading to error propagation; a wrong residual is obtained which, in turn, is used as basis for the selection of the next new support element. Thereby, the performance of the decoder is degraded.

\subsection{OMP with Soft Feedback}

We now derive a superior strategy, replacing hard quantization. Similar problems of soft-value calculation/soft feedback appear, e.g., in the fields of multiuser detection (successive interference cancellation, a.k.a. decision-feedback equalization (DFE)) [15-17].

The main idea is to replace the quantized estimates (hard decisions) inside the OMP by "soft" estimates. These should take the discrete nature of the symbols and their reliability into account, i.e., soft feedback is employed. The pseudocode representation of this strategy (denoted as SF-OMP) is also given in Alg. 1, Variant $\mathrm{C}$.

Optimally, the soft feedback for a discrete symbol $x$, given the observation $z$ and knowing the conditional probability density function (pdf) $f_{x}(x \mid z)$, is calculated as [18]

$$
\mathcal{W}(z) \stackrel{\text { def }}{=} \mathrm{E}\{x \mid z\}=\int_{-\infty}^{\infty} x f_{x}(x \mid z) \mathrm{d} x ;
$$

this calculation (carried out component-wise for a vector) replaces the hard quantization in the reconstruction algorithm (cf. Line 6 C, Alg. 1).

Modeling the problem as communication scheme, the symbol $x$ to be recovered is indirectly observed, variable $z$, through a channel. This channel, including the signal estimation step (Line 5), is given by

$$
\begin{aligned}
\boldsymbol{z} & =\boldsymbol{A}_{\mathcal{S}}^{+} \boldsymbol{y}=\boldsymbol{A}_{\mathcal{S}}^{+}(\boldsymbol{A x}+\boldsymbol{n}) \\
& =\boldsymbol{A}_{\mathcal{S}}^{+} \boldsymbol{A}_{\mathcal{S}} \boldsymbol{x}_{\mathcal{S}}+\boldsymbol{A}_{\mathcal{S}}^{+} \boldsymbol{A}_{\overline{\mathcal{S}}} \boldsymbol{x}_{\overline{\mathcal{S}}}+\boldsymbol{A} \mathcal{\mathcal { S }}^{+} \boldsymbol{n} \\
& =\boldsymbol{x}_{\mathcal{S}}+\underbrace{\left(\boldsymbol{B}_{\mathcal{S}, \mathcal{S}}\right)^{-1} \boldsymbol{B}_{\mathcal{S}, \overline{\mathcal{S}}} \boldsymbol{x}_{\overline{\mathcal{S}}}}_{\text {Interference }}+\underbrace{\boldsymbol{A}_{\mathcal{S}}^{+} \boldsymbol{n}}_{\text {Noise }} \\
& \approx \boldsymbol{x}_{\mathcal{S}}+\boldsymbol{B}_{\mathcal{S}, \overline{\mathcal{S}}} \boldsymbol{x}_{\overline{\mathcal{S}}}+\boldsymbol{A}_{\mathcal{S}}^{+} \boldsymbol{n}
\end{aligned}
$$

with the correlation matrix $\boldsymbol{B}=\left[b_{l m}\right] \stackrel{\text { def }}{=} \boldsymbol{A}^{\top} \boldsymbol{A}$, where the element $b_{l m}$ corresponds to the correlation between $\boldsymbol{a}_{l}$ and $\boldsymbol{a}_{m}$. If $\boldsymbol{A}$ is designed to have small correlation between two non-identical elements, $\boldsymbol{B}_{\mathcal{S}, \mathcal{S}}$ is close to the identity matrix and its inverse is neglected in (5). Please note, throughout the paper, the column vectors of $\boldsymbol{A}$ are assumed to be normalized to one, i.e., $b_{l l}=1, \forall l$.

The estimate $z$ consists of the already detected transmitted symbols $\boldsymbol{x}_{\mathcal{S}}$, the interference of not yet detected non-zero symbols on the symbols which have already been added to the support set (given by the second term in (4), variance $\sigma_{\mathrm{I}}^{2}$ ), and filtered channel noise $\tilde{\boldsymbol{n}} \stackrel{\text { def }}{=} \boldsymbol{A}_{\mathcal{S}}^{+} \boldsymbol{n}$. Due to the normalization of $\boldsymbol{A}$ we have $\sigma_{\tilde{\mathrm{N}}}^{2} \approx \sigma_{\mathrm{N}}^{2}$.

Hence, the error in the estimation of $z$ is caused by two different noise sources: First, the filtered AWGN generated by the channel, and second, the interference of still undetected symbols. In the following, we model the total noise (AWGN 


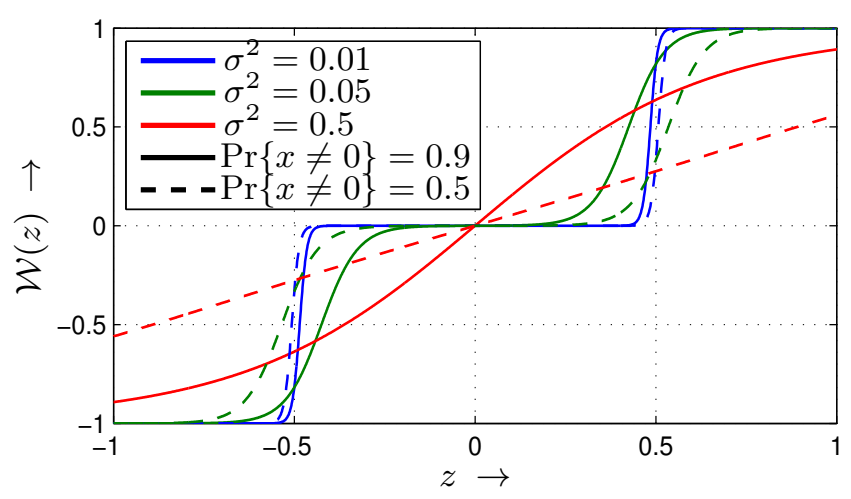

Fig. 1. Example of the soft-values $\mathcal{W}(z)$ for $\sigma^{2}=0.01,0.05,0.1$, and $\operatorname{Pr}\{x \neq 0\}=0.9$ and 0.5 .

plus interference) to be Gaussian distributed with variance $\sigma^{2} \stackrel{\text { def }}{=} \sigma_{I}^{2}+\sigma_{\mathrm{N}}^{2}$, i.e., $f_{z}(z \mid x)=\frac{1}{\sqrt{2 \pi \sigma^{2}}} \exp \left\{-(z-x)^{2} /\left(2 \sigma^{2}\right)\right\}$.

The calculation of the soft values $\mathcal{W}(z)$ for $m$-ary uniformly distributed symbols with Gaussian noise is given in [18]. However, the number of wrongly chosen elements differs from the number of correct, i.e., non-zero, elements and thus the soft values have to be calculated with respect to nonequal a priori probabilities.

Taking $f_{x}(x)=\sum_{c \in \mathcal{C}_{0}} \operatorname{Pr}\{c\} \delta(x-c)(\delta(\cdot)$ : Dirac delta function) and $f_{z}(z)=\int_{-\infty}^{\infty} f_{z}(z \mid x) f_{x}(x) \mathrm{d} x$ into account and obeying Bayes' rule [19], we have

$$
f_{x}(x \mid z)=\frac{\mathrm{e}^{-\frac{(z-x)^{2}}{2 \sigma^{2}}} \cdot \sum_{c \in \mathcal{C}_{0}} \operatorname{Pr}\{c\} \delta(x-c)}{\sum_{c \in \mathcal{C}_{0}} \operatorname{Pr}\{c\} \mathrm{e}^{-\frac{(z-c)^{2}}{2 \sigma^{2}}}} .
$$

Using this pdf in (3), the soft values calculate to

$$
\mathcal{W}(z)=\frac{\mathrm{e}^{-\frac{1}{2 \sigma^{2}}} \cdot\left(\mathrm{e}^{+\frac{z}{\sigma^{2}}}-\mathrm{e}^{-\frac{z}{\sigma^{2}}}\right)}{\mathrm{e}^{-\frac{1}{2 \sigma^{2}}} \cdot\left(\mathrm{e}^{+\frac{z}{\sigma^{2}}}+\mathrm{e}^{-\frac{z}{\sigma^{2}}}\right)+\frac{\operatorname{Pr}\{x=0\}}{\operatorname{Pr}\{x \neq 0\} / 2}} .
$$

Examples of this characteristic curve are shown in Fig. 1 for $\sigma^{2}=0.01,0.05,0.1$, and $\operatorname{Pr}\{x \neq 0\}=0.9$ (solid lines) and $\operatorname{Pr}\{x \neq 0\}=0.5$ (dashed lines). The soft values are very close to hard quantization if the variance is small (blue). The effect of the a priori probabilities is negligible in this case (solid vs. dashed). The larger the variance, the "softer" is the transition between the three states $(-1,0,+1)$ and the larger is the influence of the priors. For high variances (red), the plateau at $z=0$ vanishes and very large $|z|(\gg 1)$ are required to allow reliable decisions, i.e., $|\mathcal{W}(z)| \approx 1$.

\section{ESTIMATION OF THE PARAMETERS}

In order to calculate the soft values, the variance $\sigma^{2}$ of the total noise and the prior probabilities have to be known. Assuming that the variance $\sigma_{N}^{2}$ of the AWGN is known, we still have to estimate the interference power $\sigma_{1}^{2}$.

\subsection{Estimation of $\sigma_{1}^{2}$}

The interference $i_{l}$ affecting $x_{l}$ depends on the (yet) unknown values $x_{m}$ and on the corresponding correlation coefficients.
Let $\mathcal{S}$ denote the correct support set and $\mathcal{S}$ the current estimate within the algorithm, we have

$$
i_{l}=\sum_{m \in \mathcal{S} \backslash \mathcal{S}} b_{l m} x_{m} .
$$

Depending on the sign and on the value of the correlation between still undetected symbols and the ones we currently try to estimate, the sum interference may vary quite much.

Desired Properties of the Sensing Matrix: Before we analyze the variance of the interference, we discuss the influence of the sensing matrix $\boldsymbol{A}$ thereon. Depending on $\boldsymbol{A}$, the correlation between two vectors $\boldsymbol{a}_{l}$ and $\boldsymbol{a}_{m}$ can be quite different for different pairs, which complicates the calculation of $\sigma_{1}^{2}$ to be used for generating the soft values. Hence, a sensing matrix where the off-diagonal correlation coefficients $b_{l m}$ are as similar as possible is desirable.

In the literature, special types of matrices are known, for which the correlation between two columns is equal for all pairs, i.e., $\boldsymbol{a}_{l}^{\top} \boldsymbol{a}_{m}=b, \forall l \neq m$ [20], i.e., the Welch-bound is fulfilled with equality [21]. The vectors contained in these matrices form an equiangular tight frame (ETF) [22].

Please note that analytical constructions for ETFs are known only for specific dimensions of $\boldsymbol{A}$. A well-known construction based on so-called conference matrices is possible for $L=2 K$ and $L=p^{\alpha}+1$, where $p>2$ is a prime and $\alpha \in \mathbb{N}[20,23]$. Numerical approaches for close-tooptimal solutions are known in the literature, see, e.g., [24] and references therein.

Interference Variance of ETF Matrices: Since the actual interference (8) is not known at the decoder, an estimate that works fine for all possible cases of superposition has to be found. Numerical experiments (see also Sec. 4) reveal that it is crucial not to underestimate the interference power; the characteristic curves would be too steep pretending a too high reliability of the symbols. Consequently, we take the worst-case interference, i.e., strictly constructive superposition, into account. After the calculation of the soft estimate $\boldsymbol{w}$ (Line 6 C, Alg. 1) in the $k^{\text {th }}$ iteration, we know that approximately (on average) $\sum_{j=1}^{k}\left|w_{j}\right|$ non-zero elements have already been found. Thus,

$$
q \stackrel{\text { def }}{=} s-\sum_{j=1}^{k}\left|w_{j}\right|
$$

non-zero elements (this number may be non-integer) are missing after this iteration. The worst-case variance can then be calculated as

$$
\sigma_{\mathrm{I}_{\mathrm{Max}}}^{2}=b^{2} \cdot q^{2}
$$

where again $b=\boldsymbol{a}_{l}^{\top} \boldsymbol{a}_{m}, \forall l \neq m$.

For comparison, simulation results will also be given for the mean variance, which calculates to

$$
\sigma_{\mathrm{I}_{\text {Mean }}}^{2}=b^{2} \cdot q \text {. }
$$

The statistic of the actual interference $\left|i_{l}\right|^{2}$, cf. (8) (average over a large number of decoding runs and assuming perfect knowledge of the true support set), is shown in Fig. 2 over the iteration number $k$ of the OMP for $L=258, K=$ $129, s=20$, and $1 / \sigma_{\mathrm{N}}^{2} \widehat{=} 16 \mathrm{~dB}$. If no error occurred in the previous selection steps, the interference $i_{l}$ is binomially 


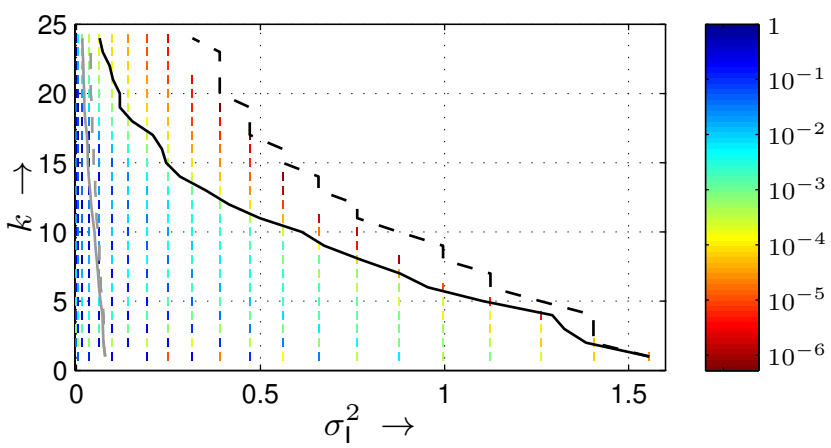

Fig. 2. Probability (color coded, averaged over 100000 decoding runs) of the actual interference power $\sigma_{1}^{2}$ over the iteration $k$ of the OMP. ETF-based matrix, $L=258, K=129, s=20$, $1 / \sigma_{\mathrm{N}}^{2} \widehat{=} 16 \mathrm{~dB} . \quad E=24 . \sigma_{\mathrm{I}_{\text {Mean }}}^{2}$ (gray lines) and $\sigma_{\mathrm{I}_{\mathrm{Max}}}^{2}$ (black lines), assuming perfect knowledge of number of missing non-zero elements (dashed lines) and estimated number $q$ (cf. (9)) (solid lines).

distributed, i.e., $\operatorname{Pr}\left\{i_{l}=j\right\}=b \cdot(1 / 2)^{s-k}\left(\begin{array}{c}s-k \\ (j+s-k) / 2\end{array}\right)$, $j=-(s-k),-(s-k)+2, \ldots, s-k$, and the interference power $\left|i_{l}\right|^{2}$ may take on squared even/odd integer multiples of $b^{2}$ if $s-k$ is even/odd.

The mean interference variance (gray) and $\sigma_{\mathrm{I}_{\operatorname{Max}}}^{2}$ (black) are also shown for reference. The solid lines show the variances with estimated number $q$ of missing elements, the dashed lines show the results assuming (genie-aided) perfect knowledge of the number of missing symbols. The mean variance (solid gray) is much too small for a number of symbols leading to an overestimation of the reliability. Instead, the worst-case interference (solid black) gives a much more conservative estimate and only truly reliable symbols are treated like hard feedback (cf. Fig. 1).

Interference Variance of General Matrices: In the case of general matrices with varying correlation coefficients, $\sigma_{\mathrm{I}_{\text {Mean }}}^{2}$ and $\sigma_{\mathrm{I}_{\mathrm{Max}}}^{2}$ depend the symbol $z_{l}, l \in \mathcal{S}$, to be estimated. The mean interference on $z_{l}$ is given by the average over all contributions by the symbols which have not yet been added to the (estimated) support set, i.e.,

$$
\sigma_{\mathrm{I}_{\text {Mean }, l}}^{2}=\frac{1}{|\overline{\mathcal{S}}|} \sum_{j \in \overline{\mathcal{S}}} b_{j l}^{2} \cdot q, \quad l \in \mathcal{S} .
$$

An upper bound on the maximal variance is given by

$$
\sigma_{\mathrm{I}_{\mathrm{Max}, l}}^{2}=\left(\frac{1}{\lfloor q\rfloor} \sum_{j=1}^{\lfloor q\rfloor} c_{j}\right)^{2} \cdot q^{2}, \quad l \in \mathcal{S},
$$

where the vector $\boldsymbol{c}$ contains the elements $\boldsymbol{b} \stackrel{\text { def }}{=}\left[\boldsymbol{B}_{l}\right]_{\mathcal{\mathcal { S }}}$ of the correlation matrix sorted according to descending magnitude. Please note that the difference between the maximal variance and the mean variance can be much larger than for ETF-based matrices. Furthermore, the variance is individual for each symbol.

\subsection{Estimation of the A Priori Probabilities}

For the calculation of the soft-values as given in (7), not only the variance of the total noise has to be known, but also the a priori probabilities of $x$.
On the one hand, it is known that the vector $\boldsymbol{x}$ has $s$ nonzero coefficients and $L-s$ zeros. On the other hand, the OMP algorithm tries to select symbols that are non-zero with high probability. Hence, the a priori probability of $x \neq 0$ for the elements in $\mathcal{S}$ is much higher than before the selection into the support set by the OMP.

However, since no knowledge about the correct priors for the elements of $\boldsymbol{x}_{\mathcal{S}}$ is available, a value has to be found that offers a good compromise for reliable vectors as well as for erroneous ones. We resort to the worst-case situation and assume a uniform distribution, i.e., $\operatorname{Pr}\{-1\}=\operatorname{Pr}\{0\}=\operatorname{Pr}\{+1\}=$ $1 / 3$. Via numerical simulations, this assumption turns out to be an almost optimum choice.

\section{SIMULATION RESULTS}

In this section, the performance of the proposed algorithm is investigated and compared to the one of OMP/Q and QOMP. Two different scenarios are considered. The upper plot in Fig. 3 shows the symbol error rate (SER) over the noise level in $\mathrm{dB}$ for an ETF-based sensing matrix (i.e., equal correlation for all basis vectors) taken from [23] with $L=258$, $K=129$ and $s=20$. For comparison, the performance for random matrices is also shown in Fig. 3 (bottom). In this case, the sensing matrix is a randomly chosen $K \times L$ dimensional part of a random unitary matrix. In both scenarios, the columns of $\boldsymbol{A}$ are normalized to one.

In order to achieve optimal results, the OMP carries out $E=24$ iterations, although the sparsity is $s=20[7,13]$. The threshold for the hard quantization inside the Q-OMP is optimized to 0.6 . The quantization threshold at the end of the algorithms is adjusted such that $\hat{\boldsymbol{x}}$ has the desired sparsity.

While hard quantization inside the OMP (Q-OMP, green) has a small gain for high noise levels compared to the conventional OMP with successive quantization (Q/OMP, red), Q-OMP performs even worse than OMP/Q for low noise levels in both scenarios.

OMP with soft feedback and perfect knowledge of both the exact actual variance (cf. (4)) and the priors (SF-OMP, black), however, clearly outperforms both previously mentioned algorithms. Since the loss by using the approximated variances (purple, cf. (5)) is negligible, all further evaluations are done for these simplified equations. These results with genie-aided knowledge can be regarded as bounds for the achievable performance.

While no loss can be observed if $\sigma_{\mathrm{I}_{\text {Max }}}^{2}$ (turquois) is used instead of the genie-aided correct interference (purple) in the case of ETF-based matrices (top), the performance decreases for random matrices since the worst-case estimation of the interference is degraded by elements with very high correlation. Please note that the usage of $\sigma_{\mathrm{I}_{\text {Mean }}}^{2}$ (yellow) clearly degrades the performance such that there is no benefit compared to hard quantization (green). This is due to the fact that $\sigma_{\mathrm{I}_{\text {Mean }}}^{2}$ is smaller than the actual variance for some symbols (cf. Fig. 2) and thus the soft values of these unreliable symbols are effectively equal to the ones of hard quantization.

If - in addition to the variance-also the priors are not perfectly known (blue vs. turquois), the performance decreases 
for very small noise levels if $\sigma_{\mathrm{I}_{\mathrm{Max}}}^{2}$ is employed. In this case, the selection of new elements by the OMP is very reliable and hence only very few vectors benefit from the high uncertainty introduced by equal priors, whereas most vectors suffer from the artificial unreliability. For $\sigma_{\mathrm{I}_{\text {Mean }}}^{2}$ (orange), the performance is again comparable to the one of Q-OMP.

Summing up, SF-OMP (blue) clearly outperforms OMP/Q (red) and Q-OMP (green) by about $1 \mathrm{~dB}$ in the high-SNR regime. By using $\sigma_{\mathrm{I}_{\mathrm{Max}}}^{2}$ and $\operatorname{Pr}\{0\}=1 / 3$, there is only a small loss compared to decoding with perfect knowledge. The results for $\sigma_{\mathrm{I}_{\text {Mean }}}^{2}$ are not better than for Q-OMP. In general, random matrices perform worse than ETF-based.
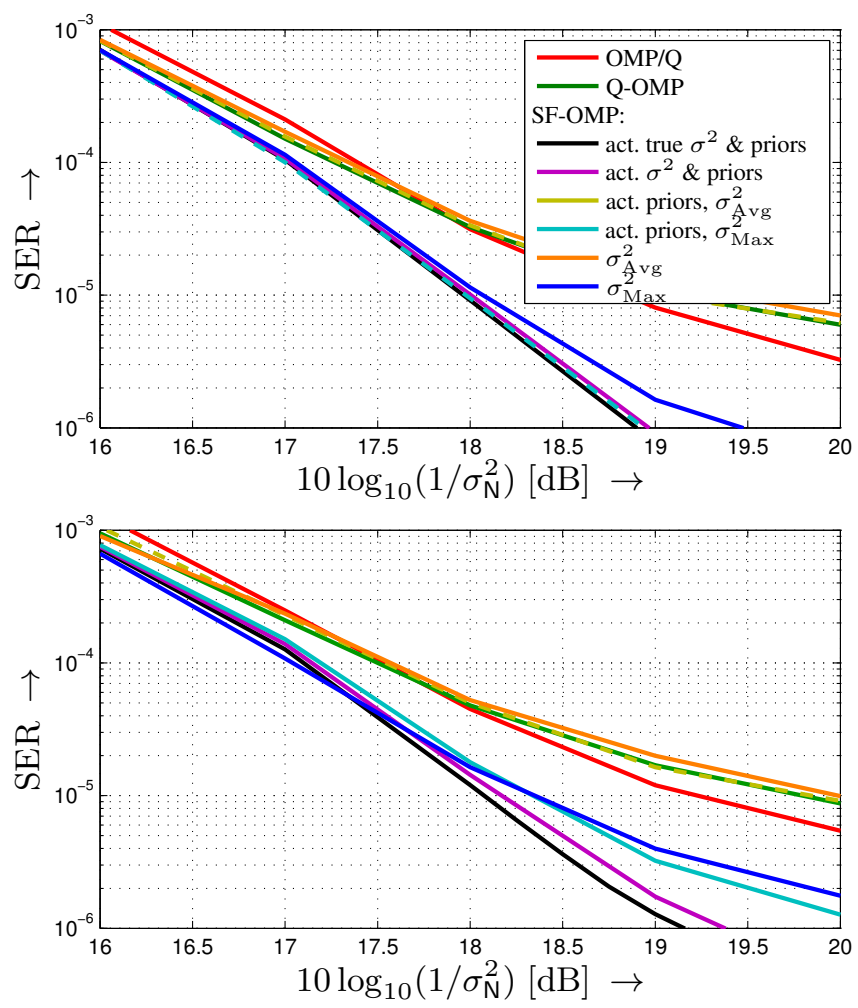

Fig. 3. SER of the proposed variants of OMP over the noise level $1 / \sigma_{n}^{2}$ in dB. $L=258, K=129, s=20, \mathcal{C}=\{-1,+1\}$. ETFbased matrix (top), random matrix (bottom). $E=s+4$.

\section{CONCLUSION}

In this paper, we have proposed a new algorithm for the reconstruction of discrete-valued sparse signals. The well-known OMP has been modified to incorporate the knowledge about the discrete nature of $\boldsymbol{x}$ and the actual noise level. Therefore, soft-values are calculated in each iteration, followed by a quantization step at the end of the algorithm.

The performance of the proposed approach clearly outperforms the state-of-the-art solutions. When using an optimized sensing matrix, there is only a very small loss compared to reconstruction with (genie-aided) perfect knowledge.

\section{REFERENCES}

[1] H. Zhu, G.B. Giannakis. Exploiting Sparse User Activity in Multiuser Detection. IEEE Tr. Comm., pp. 454-465, Feb. 2011.

[2] R.F.H. Fischer, F. Wäckerle. Peak-to-Average Power Ratio Reduction in OFDM via Sparse Signals: Transmitter-Side Tone Reservation vs. Receiver-Side Compressed. Proc. Int. OFDM Workshop, Essen, Germany, Aug. 2012.

[3] A. Ens, A. Yousaf, T. Ostertag L.M. Reindl. Optimized Sinus Wave generation with Compressed Sensing for Radar Applications. Proc. CoSeRa, Bonn, Germany, Sept. 2013.

[4] P. Dymarski, R. Romaniuk. Sparse Signal Modeling in a Scalable CELP Coder. In Proc. EUSIPCO 2013, Marrakech, Morocco, Sep. 2013.

[5] D.L. Donoho. Compressed Sensing. IEEE Tr. Inf. Theory, pp. 1289-1306, 2006.

[6] G.L. Nehmhauser, L.A. Wolsey. Integer and Combinatorial Optimization, John Wiley \& Sons, New York, 1988.

[7] S. Sparrer, R.F.H. Fischer. Adapting Compressed Sensing Algorithms to Discrete Sparse Signals. Proc. Workshop on Smart Antennas (WSA) 2014, Erlangen, Germany, Mar. 2014.

[8] E. Agrell, T. Eriksson, A. Vardy, K. Zeger. Closest Point Search in Lattices. IEEE Tr. Inf. Theory, pp. 2201-2214, Aug. 2002.

[9] Y.C. Pati, R. Rezaiifar, P.S. Krishnaprasad. Orthogonal Matching Pursuit: Recursive Function Approximation with Applications to Wavelet Decomposition. Proc. Asilomar Conf., pp. 4044, Nov. 1993.

[10] D. Needell, J.A. Tropp. CoSaMP: Iterative Signal Recovery from Incomplete and Inaccurate Samples. Applied and Comp. Harm. Analysis, pp. 301-321, May 2009.

[11] A. Cohen, W. Dahmen, R. DeVore. Instance Optimal Decoding by Thresholding in Compressed Sensing. Proc. Int. Conf. on Harm. Anal. and Part. Diff. Equ., Madrid, Spain, June 2008.

[12] S.D. Babacan, P. Molina, A.K. Katsaggelos. Bayesian Compressive Sensing Using Laplace Priors. IEEE Tr. Image Proc., pp. 53-63, Jan. 2010.

[13] T. Zhang. Sparse Recovery With Orthogonal Matching Pursuit Under RIP. IEEE Tr. Inf. Theory, pp. 6215-6221, Sept. 2011.

[14] S. Sparrer, R.F.H. Fischer. Discrete Sparse Signals: Compressed Sensing by Combining OMP and the Sphere Decoder. arXiv, 1310.2456, 2013.

[15] T. Frey, M. Reinhardt. Signal Estimation for Interference Cancellation and Decision Feedback Equalization. Proc. IEEE Vehicular Techn. Conf., pp. 113-121, Phoenix, USA, May 1997.

[16] A. Lampe, J.B. Huber. On Improved Multiuser Detection with Iterated Soft Decision Interference Cancellation. Proc. Comm. Theory Mini-Conf. at GLOBECOM, pp. 172-176, June 1999.

[17] R.R. Müller, J.B. Huber. Iterated Soft-Decision Interference Cancellation for CDMA. Broadband Wireless Comm., eds. M. Luise and S. Pupolin, pp. 110-115, Springer London, 1998.

[18] A. Engelhart, W.G. Teich, J. Linder. Complex Valued Signal Estimation for Interference Cancellation Schemes. Tech. Rep. ITUU-TR-1998/04, Dept. of Inf. Tech., Uni Ulm, Dec. 1998.

[19] A. Papoulis, S.U. Pillai. Probability, Random Variables and Stochastic Processes. McGraw-Hill, 4th edition, 2002.

[20] J.H. Conway, R.H. Hardin, N.J.A. Sloane. Packing Lines, Planes, etc.: Packings in Grassmannian Spaces. Experimental Mathematics, pp. 139-159, Jan. 1996.

[21] L. Welch. Lower Bounds on the Maximum Cross Correlation of Signals. IEEE Tr. Inf. Theory, pp. 397-399, 1974.

[22] P.G. Casazza, G. Kutyniok, F. Philipp. Introduction to Finite Frame Theory. Finite Frames, pp. 1-53, Birkhäuser, 2013.

[23] R.W. Heath, T. Strohmer, A.J. Paulraj. Grassmannian Signatures for CDMA Systems. Proc. GLOBECOM, pp. 1553-1557, San Francisco, USA, 2003.

[24] H. Zörlein, M. Bossert. Coherence Optimization and Best Complex Antipodal Spherical Codes. arXiv, 1404.5889, 23. Apr. 2014. 